\title{
Aneurysm of the Superior Posterior Pancreatic-Duodenal Artery Presenting with Recurrent Syncopes
}

\author{
I.A.J. van Doesburg D. Boerma M. van Leersum \\ B. van Ramshorst \\ St Antonius Hospital, Department of Surgery, Nieuwegein, The Netherlands
}

\section{Key Words}

Pancreatic bleeding $\cdot$ Pancreatic haemorrhage $\cdot$ Pancreaticorrhagia $\cdot$ Pancreatic-duodenal artery $\cdot$ Pancreatic aneurysm $\cdot$ Aneurysm

\begin{abstract}
We present a 61-year-old woman with hypovolemic shock due to a ruptured aneurysm of the superior posterior pancreatic-duodenal artery in whom recurrent syncopes were the first presenting sign of pancreatic-duodenal artery aneurysm (PDAA). PDAA is a rare but life-threatening condition. The widely varying symptomatology may lead to a delay in diagnosis and treatment. Patients with atypical symptoms, such as vague abdominal pain, recurrent dizziness or syncope, may actually suffer from a sentinel bleeding of the vascular malformation. Radiological imaging, especially selective angiography, may provide a diagnostic as well as a therapeutic tool in these patients.
\end{abstract}

\section{Introduction}

Pancreatic-duodenal artery aneurysms (PDAAs) are rare and represent $2 \%$ of all visceral artery aneurysms [1-5]. Since 1895, about 100 cases have been reported in the literature, mostly as individual case reports $[2,6]$. The incidence, however, is rising due to the increasing use of imaging techniques [5]. PDAAs often remain asymptomatic and are often diagnosed incidentally or in the course of the investigation of unspecified abdominal complaints [2]. They can be distinguished into true aneurysms, which are associated with celiac axis stenosis, and false aneurysms, which are thought to be related to pancreatitis, septic emboli and trauma $[1-5,7-8]$. We present a case of intraabdominal haemorrhage due to a ruptured aneurysm of the superior posterior pancreatic-duodenal artery in a 61-year-old woman in whom recurrent events of syncope were the first 
presenting signs. The clinical presentation, aetiology and therapeutic options are discussed.

\section{Case Report}

A 61-year-old woman, with no relevant medical history, consulted her general practitioner because of recurrent episodes of syncope. After evaluation she was referred to and admitted by a neurologist. The day before admission the patient experienced an episode of vague abdominal pain. At the fourth day of admission, a new episode of syncope occurred during which the patient became progressively hypotensive, not responding to intravenous fluid therapy. Blood tests showed anaemia (haemoglobin $4.7 \mathrm{mmol} / \mathrm{l}$, haematocrit $22 \%$ ).

An emergency left subcostal laparotomy was performed under the diagnosis of a ruptured spleen. A pulsating retroperitoneal haematoma was found, upon which the patient was transferred to our hospital. CT angiography scan showed an extravasation of one of the branches of the superior mesenteric artery and a large haematoma around the duodenum (fig. 1). Subsequent coiling by selective angiography (fig. 2) unfortunately was unsuccessful, due to small diameter and tortuosity of the ruptured vessel. Because of ongoing haemodynamic instability, an emergency midline laparotomy was performed. At operation a large retroperitoneal haematoma was found, shifting the right kidney and hemicolon to the midline. Two liters of free blood was evacuated. There was no ongoing haemorrhage, and as the exact source of the bleeding could not be identified, the patient's abdomen was packed. Following surgery the patient stabilized haemodynamically. At second-look laparotomy two days later a ruptured aneurysm of the superior posterior pancreatic-duodenal artery was identified as the bleeding focus. The artery was ligated proximally and distally to the bleeding source. Further recovery was uneventful.

\section{Discussion}

The presentation of a PDAA can vary widely and diagnosis can be difficult, resulting in significant delay in treatment. Most PDAAs remain asymptomatic and are discovered incidentally on CT scan or angiography during the investigation of an unrelated illness [2]. Symptomatic PDAAs may present with a wide range of symptoms, of which abdominal pain and hypovolemic shock due to rupture and intraabdominal haemorrhage are the most common $[2,4,5,9]$.

In our patient the rupture of the aneurysm was initially contained by the pancreatic tissue, a so-called 'sentinel bleed', causing a short period of hypotension leading to syncope. This case once again displays the importance of imaging techniques in the management of a haemodynamically unstable patient suspected of intraabdominal haemorrhage. Prior to the patient's first laparotomy, no attempt was made to identify the source of the bleeding using imaging techniques nor to perform an endovascular intervention. Nowadays, preoperative imaging by CTA and/or (selective) angiography is mandatory in the work-up of haemodynamically instable patients. In our patient both imaging techniques showed a bleeding in a branch of the superior mesenteric artery, although none of the investigations in our case specifically showed a PDAA. Computed angiographic tomography or selective angiography are the main imaging techniques to detect a PDAA [4].

As most PDAA patients present acutely with rupture, a high mortality rate of $20-30 \%$ has been described $[2,3,8]$. At present, embolization of PDAAs is considered the treatment of choice for both ruptured and nonruptured aneurysms [2, 5]. Endovascular treatment includes obliteration of the aneurysm using embolization agents [2]. Better resuscitation methods and the use of catheter embolization have reduced mortality over the past decades [3]. 


\begin{tabular}{r|l|l|l} 
Case Reports $/$ h & $\begin{array}{l}\text { Case Rep Gastroenterol 2009;3:230-234 } \\
\text { D01: 10.1159/000227735 }\end{array}$ & Published online: August 28, 2009 & $\begin{array}{l}\text { O 2009 S. Karger AG, Basel } \\
\text { ISSN 1662-0631 } \\
\text { www.karger.com/crg }\end{array}$ \\
\hline
\end{tabular}

The aetiology of the PDAA in this patient remains unclear. She had not suffered abdominal trauma, and a normal celiac axis was demonstrated at CT angiography. In the absence of a history of trauma or pancreatitis, it is most likely that this patient had a true aneurysm. Only one similar case of PDAA with unknown aetiology has been described in the literature before [2].

In conclusion, PDAA is a rare but clinically significant condition which can present with vague symptoms that may end up in a life-threatening situation, due to a delay in diagnosis and treatment. The initial presentation of the PDAA in our patient with recurrent syncopes has not been described before. Although generally accepted, this case confirms that imaging techniques (CT or selective angiography) are essential in a patient suspected of ongoing intraabdominal haemorrhage. Even when surgical exploration is tempting, every attempt to first perform an angiographic examination with subsequent endovascular intervention should be made.

Fig. 1. An active extravasation of contrast arising from a branch of the superior mesenteric artery with a large haematoma around the duodenum.

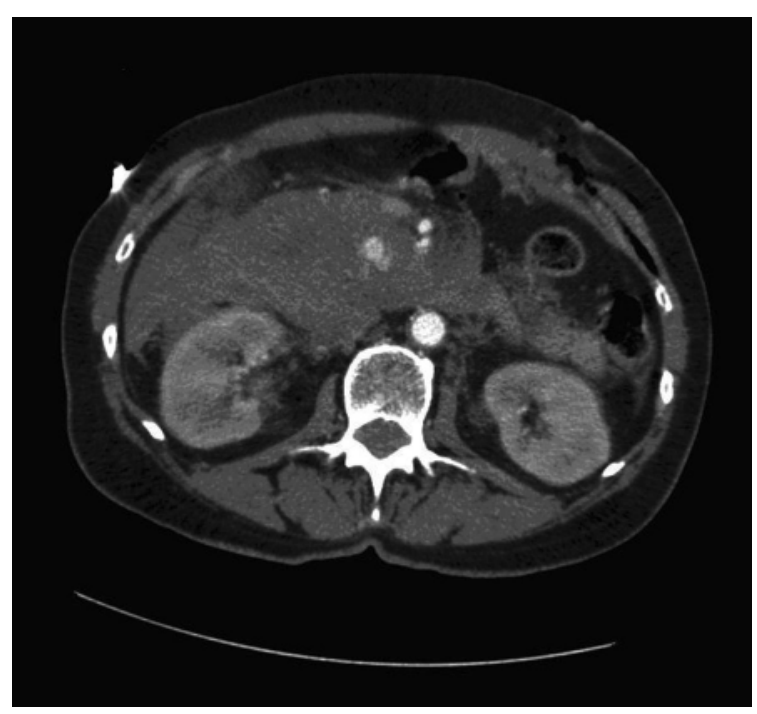


Fig. 2. Selective angiography of the superior mesenteric artery. Approximately $5 \mathrm{~cm}$ from the major branch a small bleeding infarction is visible. Also, a large connection between the superior mesenteric artery and the celiac trunk via the gastroduodenal artery is present. This connection is also feeding the small bleeding.

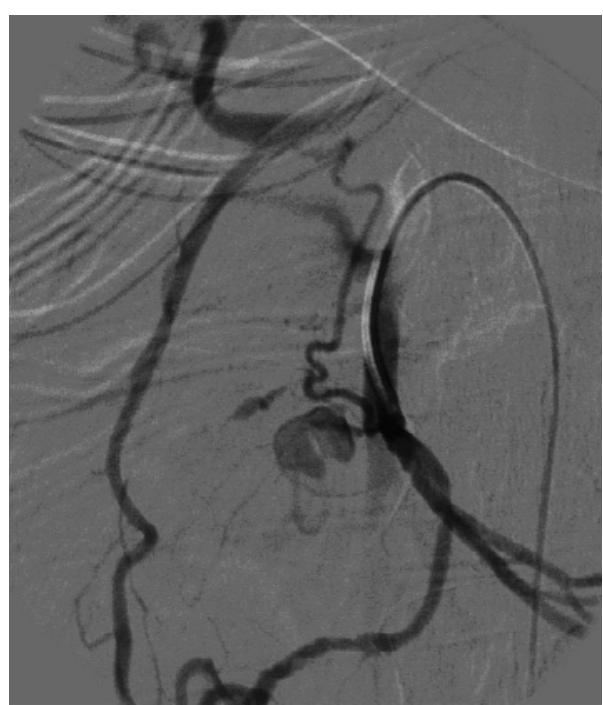




\section{References}

1 Stabile BE, Wilson SE, Debas HT: Reduced mortality from bleeding pseudocysts and pseudoaneurysms caused by pancreatitis. Arch Surg 1983;118:45-51.

2 Sharif MA, O’Donnell ME, Johnston LC, Lau LL: Successful surgical management of a ruptured true pancreaticoduodenal artery aneurysm following failed transcatheter embolization. Vascular 2007;15:231-234.

-3 de Perrot M, Berney T, Deléaval J, Bühler L, Mentha G, Morel P: Management of true aneurysms of the pancreaticoduodenal arteries. Ann Surg 1999;229:416-420.

-4 Eckhauser FE, Stanley JC, Zelenock GB, Borlaza GS, Freier DT, Lindenauer SM: Gastroduodenal and pancreaticoduodenal artery aneurysms: a complication of pancreatitis causing spontaneous gastrointestinal hemorrhage. Surgery 1980;88:335-344.

-5 Quandalle P, Chambon JP, Marache P, Saudemont A, Maes B: Pancreaticoduodenal artery aneurysms associated with celiac axis stenosis: report of two cases and review of the literature. Ann Vasc Surg 1990;4:540-545.

6 Ferguson F: Aneurysm of the superior pancreaticoduodenal artery. Proc N Y Pathol Soc 1895;24:45-49.

7 de Moya MA, Reisner AT, LaMuraglia GM, Kalva SP: Case 1-2008. A 45-year-old man with sudden onset of abdominal pain and hypotension. N Engl J Med 2008;358:178-186.

8 Stanley JC, Whitehouse WM: Splanchic artery aneurysms; in Rutherford RB (ed): Vascular Surgery. Philedelphia, WB Saunders, 1984, pp 798-813.

-9 Uher P, Nyman U, Ivancev K, Lindh M: Aneurysms of the pancreaticoduodenal artery associated with occlusion of the celiac artery. Abdom Imaging 1995;20:470-473. 